\title{
TEM Characterization of the Deformed Region Beneath Knoop Indents in Boron Carbide
}

\author{
S. D. Walck ${ }^{1}$, J. C. LaSalvia ${ }^{2}$, and K. D. Behler ${ }^{3}$ \\ 1. TKC Global, RDRL-WMM-C, Aberdeen Proving Ground, MD 21005, USA \\ 2. U.S. Army Research Laboratory, RDRL-WMM-E, Aberdeen Proving Ground, MD 21005, USA \\ 3. TKC Global, RDRL-WMM-E, Aberdeen Proving Ground, MD 21005, USA
}

Understanding the deformation mechanisms in ceramic materials is crucial for developing and optimizing next-generation ceramic materials in body and vehicle armor systems. In an effort to better understand the mechanistic response of polycrystalline boron carbide to large contact stresses, transmission electron microscopy (TEM) methods were used to examine thin cross-sections of the inelastically deformed regions beneath Knoop indents of various loads and load-dwell times. Indentation allows the study of deformation of microstructural features as a function of distance and depth from the loading and allows for comparison to mechanistic modeling. TEM cross-sections were prepared from 0.3 , 1, and $2 \mathrm{kgf}$ Knoop indents in a hot-pressed polycrystalline boron carbide. Due to excessive spallation of material surrounding $2 \mathrm{kgf}$ indents, load-dwell times of 15 and $45 \mathrm{~s}$ were only used for the 0.3 and $1 \mathrm{kgf}$ indent loads. Although FIB preparation of nano-indentation methods have been used to examine the areas under indents with great success [1], the higher loads applied in this study allows a more comprehensive examination of the nature of the sub-surface inelastic region and the extended cracking. However, the extensive cracking presents problems for sample preparations that were solved by Brennan et al. using a multi-step sample preparation approach [2]. For the work in this study, an improved technique that was less labor intensive was used [3]. The process involves first preparing the indents in an appropriately spaced array across the surface near the edge, sufficiently close to use a masked-ion milling system (MIMS), but not close enough that the fracture mechanics were compromised. The cross sections of the indents were examined and indexed in an SEM, as shown in Fig 1, and then vacuum infiltrated with a low viscosity epoxy in order to fill the open cracks. The TEM samples were then prepared by in-situ lift-out technique after specific indents were located in the FIB by using the index distances found previously.

TEM characterization of the inelastically deformed regions showed extensive stress-induced amorphization, micro-cracking, and macro-cracking. Observations clearly show that these deformation mechanisms are related. Stress-induced amorphization predominately occurred in discrete slip bands that were nanoscale in width and microscale in length. Micro-cracks were observed at slip band intersections, while macro-cracking appeared to be a consequence of both mechanisms. The trajectory of slip bands appeared similar to slip lines for blunt-wedge indentation, indicating the importance of shear stress on their formation.

\section{References:}

[1] K. Madhav Reddy et al., Nature Communications 10.1038 (2013) p. 3483.

[2] C.V Brennan, S.D Walck, and J. J. Swab, Microscopy and Microanalysis 20(6) (2014), p. 1646.

[3] J. J. Swab, et al., Microscopy and Microanalysis 21(S3), (2015) p. 315.

[4] The research reported in this document was performed in connection with contract/instrument

W911QX-14-C-0016 with the U.S. Army Research Laboratory. The views and conclusions contained in this document are those of TKC Global and the U.S. Army Research Laboratory. 


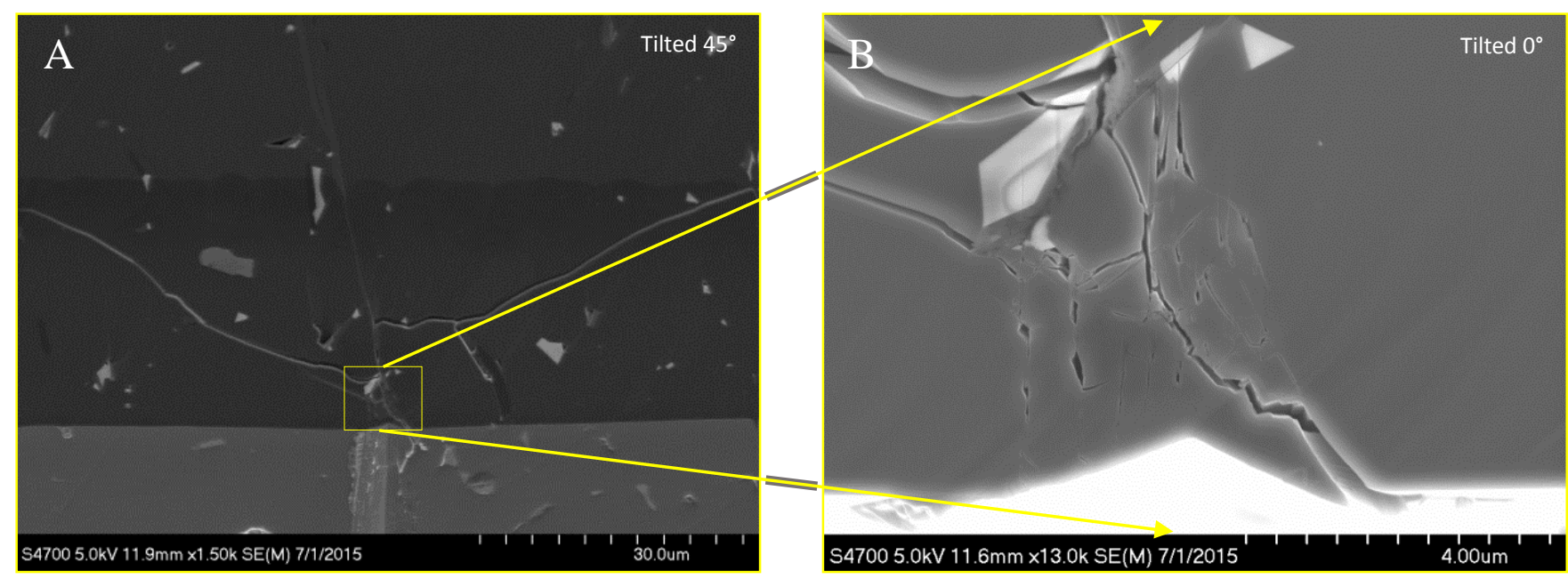

Figure 1 A)SEM image of MIMS cut sample tilted at $45^{\circ}$ showing the cross section and surface of a $1 \mathrm{kgf}$ Knoop indent. B) Enlarged area of (A) showing sub-surface cracking under the indent.

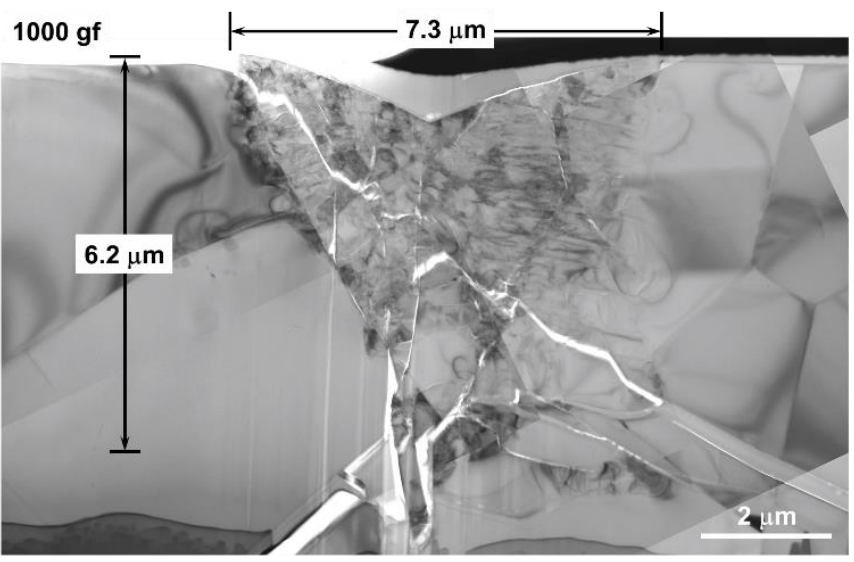

Planar Defects, Amorphous Bands, Microcracks, and Macrocracks

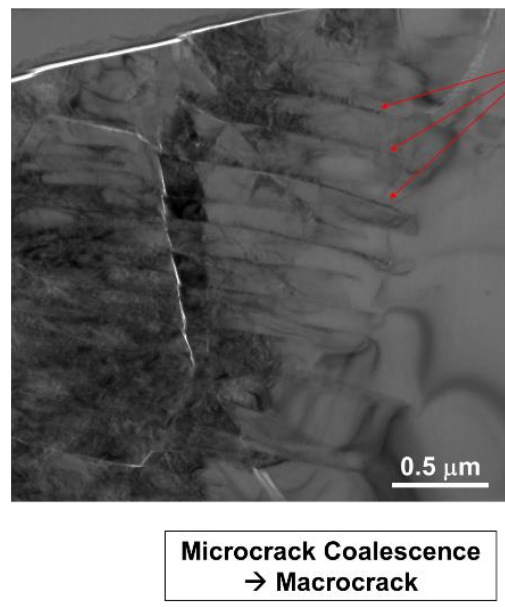

$\rightarrow$ Macrocrack

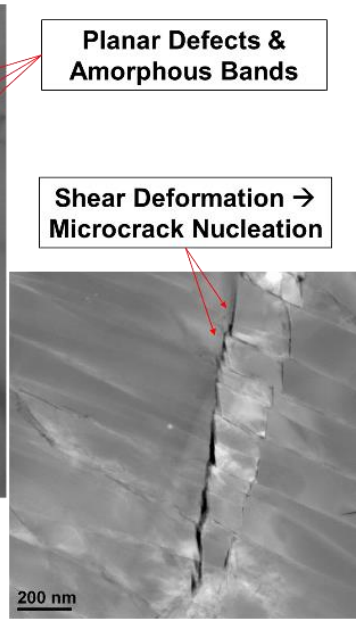

Figure 2 A) Low Mag XTEM Montage image of $1 \mathrm{kgf}$ Knoop indent. B) Enlarged area from (A) showing micro and macro crack formation within the inelastic region under the indent.
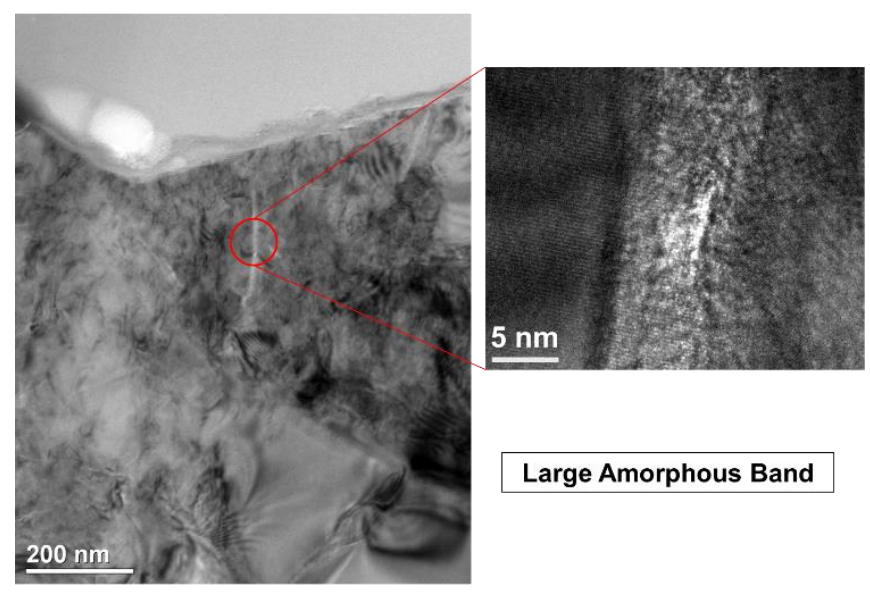

Large Amorphous Band

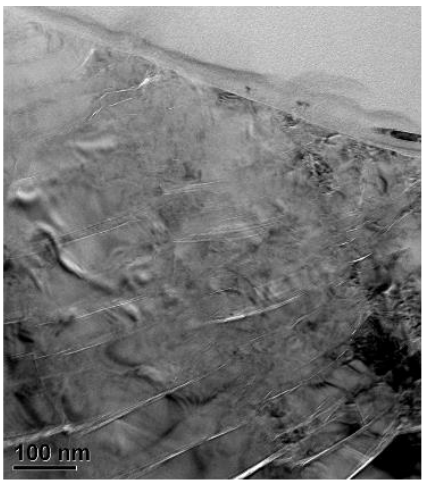

Amorphous Band Orientations Nominally Parallel to Maximum Shear Stress Directions

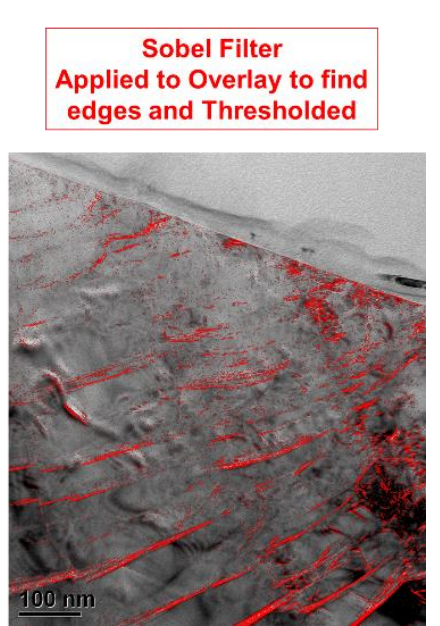

Figure 3 Inelastic region under a $1 \mathrm{kgf}$ Knoop the indent showing the presence and distribution of amorphous shear bands. 